\title{
Competition between spin, charge, and bond waves in a Peierls-Hubbard model
}

\author{
Pablo A. Venegas \\ Departamento de Física, Faculdade de Ciências, Universidade Estadual Paulista, Campus Bauru, Av. Engenheiro Luis Coube s/n, \\ 17033-360 Bauru, São Paulo, Brazil \\ Carlos Henríquez \\ Comisión Chilena de Energía Nuclear, Casilla 118-D, Santiago, Chile \\ Jaime Rössler \\ Departamento de Física, Facultad de Ciencias, Universidad de Chile, Casilla 653, Santiago, Chile
}

(Received 26 March 1996)

\begin{abstract}
We study a one-dimensional extended Peierls-Hubbard model coupled to intracell and intercell phonons for a half-filled band. The calculations are made using the Hartree-Fock and adiabatic approximations for arbitrary temperature. In addition to static spin, charge, and bond density waves, we predict intermediate phases that lack inversion symmetry, and phase transitions that reduce symmetry on increasing temperature. [S0163-1829(96)04229-4]
\end{abstract}

\section{INTRODUCTION}

One-dimensional electronic systems have been extensively studied in the literature over the last two decades. ${ }^{1-14}$ In order to obtain a theoretical understanding of them, two main aspects have been considered: electron-electron $(e-e)$ repulsion $^{3-5}$ and electron-phonon ( $e$-ph) coupling. ${ }^{6-8}$ Also, the combined effect of both interactions has been studied. ${ }^{9-13}$ Due to these interactions, an ordered phase is expected at zero temperature, with a $2 k_{F}$ or $4 k_{F}$ modulation of the lattice. However, quantum fluctuations may reduce the former effect to short-range correlations. ${ }^{6-8}$

In the particular case of a half-filled band, the lattice and distortion periods are commensurable by the simple rational $\frac{1}{2}$. Therefore quantum fluctuations are strongly suppressed, as the period-2 modulation is hard locked to the lattice. ${ }^{6,13}$ The last one is supported by Monte Carlo simulations, 7,10 where distortions with long-range order (LRO) are reported for realistic (not too high) phonon frequencies. Since the half-filled band chain is an insulator, three main ordered phases can appear: a spin density wave $(\mathrm{SW}){ }^{9}$ a charge density wave $(\mathrm{CW}),{ }^{1,13}$ or a bond density wave $(\mathrm{BW}) ;^{1,12}$ these phases can also coexist. ${ }^{1,9,13}$

The present paper deals with a half-filled band extended

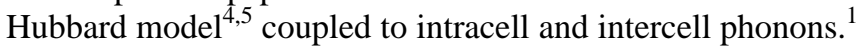
The analysis is performed for arbitrary temperature. We use Hartree-Fock (HF) (Refs. 1,9,12) and adiabatic approximations; consequently a period-2 static deformation is expected. The adiabatic approximation yields a small overestimation of Peierls distortion (e.g., a $15 \%$ in polyacetylene ${ }^{7}$ ). Despite the HF limitations, ${ }^{14}$ in the present case (where a finite gap separates the filled and empty electronic states) HF becomes fair, and its predictions over some actual systems are in good agreement with experiments. ${ }^{12}$

Rössler and Gottlieb ${ }^{1}(\mathrm{RG})$ analyzed a model similar to the present one, although they considered a 1/4-filled band and an infinite intracell Coulomb repulsion. They used a mean field approach, obtaining results formally equivalent to the present ones by a substitution of parameters. Other authors have also faced similar, but rather simpler models, ${ }^{2,7,10,13}$ analyzing the $T=0$ case.

Our generalized Peierls-Hubbard Hamiltonian is

$$
\begin{aligned}
H= & -\sum_{l, \sigma}\left[t-g\left(u_{l+1}-u_{l}\right)\right]\left(c_{l \sigma}^{\dagger} c_{l+1, \sigma}+c_{l+1, \sigma}^{\dagger} c_{l, \sigma}\right) \\
& +\sum_{l, \sigma} \check{n}_{l, \sigma}\left[-\lambda v_{l}+\frac{U}{2} \check{n}_{l,-\sigma}+V \sum_{\sigma^{\prime}} \check{n}_{l+1, \sigma^{\prime}}\right] \\
& +\frac{1}{2} \sum_{l}\left[K\left(u_{l+1}-u_{l}\right)^{2}+Q v_{l}^{2}\right]
\end{aligned}
$$

here $v_{l}$ and $u_{l}$ represent intracell and intercell displacements respectively, $c_{l, \sigma}^{\dagger}$ creates a spin $\sigma$ electron on site $l$, and $\check{n}_{l, \sigma} \equiv c_{l, \sigma}^{\dagger} c_{l, \sigma}$. We choose $t=1$ as the energy unit.

Applying the HF approximation to the $e-e$ interactions and using the period-2 translational symmetry in the electronic averages, we have $\left\langle\check{n}_{l, \sigma}\right\rangle \equiv \frac{1}{2}+(-1)^{l} \Gamma_{\sigma}$. Due to Mattis's theorem, the spin up and down directions are equivalent in a one-dimensional system. Thus, choosing $\Gamma_{\uparrow} \equiv \Gamma \geqslant 0$ we have $\Gamma_{\downarrow}=\Gamma$ for a $\mathrm{CW}$, while $\Gamma_{\downarrow}=-\Gamma$ for a SW. In addition $\left\langle c_{l, \sigma}^{\dagger} c_{l+1, \sigma}\right\rangle \equiv \tau+(-1)^{l} \Delta$.

The equilibrium values of $\left\{u_{l}, v_{l}\right\}$ are obtained by minimizing the Helmholtz free energy. Using the HellmannFeynman theorem, we conclude that $u_{l+1}-u_{l}=-(4 g /$ $K)\left[\tau+(-1)^{l} \Delta\right]$ and $v_{l}=(\lambda / Q)\left[1+(-1)^{l}\left(\Gamma+\Gamma_{\downarrow}\right)\right]$. Thus, $\Delta$ measures the fluctuations in intercell distance associated with a BW phase. We note that $\Gamma \leqslant \frac{1}{2}$ and $\Delta \leqslant \frac{1}{4}$. The case $\Gamma=\frac{1}{2}$ represents a saturated $\mathrm{CW}$ or SW, while $\Delta=\frac{1}{4}=\tau$ corresponds to a saturated $\mathrm{BW}$, where the system breaks into N/2 dimers. ${ }^{1}$ The SW phase produces a uniform lattice contraction, instead of a Peierls distortion.

Later developments transform our Hamiltonian into the "effective", one 


$$
\begin{aligned}
H_{\mathrm{eff}}= & -A \Gamma \sum_{l, \sigma}(-1)^{l} \theta_{\sigma} \check{n}_{l, \sigma}-\sum_{l, \sigma}\left[W+G(-1)^{l} \Delta\right] \\
& \times\left[c_{l \sigma}^{\dagger} c_{l+1, \sigma}+c_{l+1, \sigma}^{\dagger} c_{l, \sigma}\right],
\end{aligned}
$$

where $A=U \equiv A_{\mathrm{SW}}$ for SW, $A=4 V-U+2 \lambda^{2} / Q \equiv A_{\mathrm{CW}}$ for $\mathrm{CW}, W=t+G \tau$, and $G=V+4 g^{2} / K$. The parameter $W$ is an "effective" electronic transfer term, where $t$ is corrected due to lattice contraction and HF exchange contribution.

We diagonalize $H_{\text {eff }}$ obtaining its quasiparticle spectrum

$$
W_{k, \xi}=\xi \sqrt{4 W^{2} \cos ^{2} k+4 G^{2} \Delta^{2} \sin ^{2} k+A^{2} \Gamma^{2}},
$$

with $\xi= \pm$.

Here $-\frac{1}{2} \pi<k \leqslant \frac{1}{2} \pi$ is the new Brillouin zone. The relation $W_{k} \equiv W_{k,+}=-W_{k,-}$ implies a zero chemical potential. We evaluate the electronic averages using the eigenfunctions of $H_{\text {eff }}$. The latter yields the following self-consistent conditions for $\tau$ and the order parameters $\Gamma, \Delta$ :

$$
\left\{\begin{array}{c}
\Gamma \\
\Delta \\
\tau
\end{array}\right\}=\frac{2}{N} \sum_{k=0}^{\pi / 2} \frac{1}{W_{k}} \tanh \left(\frac{W_{k}}{2 T}\right)\left\{\begin{array}{c}
A \Gamma \\
2 G \Delta \sin ^{2}(k) \\
2 W \cos ^{2}(k)
\end{array}\right\} .
$$

These conditions coincide with those of RG under the substitutions $V \rightarrow A, G_{2}=0, \Delta_{1} \rightarrow \Delta$ and $G_{1} \rightarrow G$; here the left side corresponds to RG.

After some calculations we also obtain the Helmholtz free energy $F=2 G\left[\tau^{2}+\Delta^{2}\right]+A \Gamma^{2}-(8 T /$ N) $\sum_{k=0}^{\pi / 2} \ln \left[2 \cosh \left(W_{k} / 2 T\right)\right]$.

We use $F$ to determine the most stable phase at a given temperature. It is easy to prove that $\partial F / \partial A=-\Gamma^{2} \leqslant 0$. Therefore, defining $V_{\text {eff }}=V+\lambda^{2} /(2 Q)$, we have

$$
A=\max \left\{A_{\mathrm{SW}}, A_{\mathrm{CW}}\right\}=2 V_{\mathrm{eff}}+\left|U-2 V_{\mathrm{eff}}\right| .
$$

Thus, the SW phase is stable if $U>2 V_{\text {eff }}$ and vice versa for the $\mathrm{CW}$ phase. Monte Carlo simulations on a rigid lattice ${ }^{5}$ give a very small departure from the latter condition.

We define $D=A-2 G$ and use the replacements specified below Eq. (4) in order to describe our system in terms of the two parameters of RG ( $D$ and $G$ ), thus inheriting their results. However, since the present model differs from RG, the physical conclusions are also distinct. In particular, the CW phase of RG becomes our SW phase if $U>2 V_{\text {eff }}$. In this way, the seven parameters appearing in our Hamiltonian (1) reduce to two independent constants, plus the sign of $U-2 V_{\text {eff }}$ (remember that we chose $t=1$ ). Moreover, for a pure BW phase (case $\Gamma=0$ ) the parameter $D$ does not play any role, excepting for determining the BW boundary.

It is useful to define $L \equiv 4 g^{2} / K-\lambda^{2} /(2 Q)$; with $L$ as a measure of the competition between the intercell and intracell $e$-ph interactions. Now $G=L+V_{\text {eff }}$ and $D=\left|U-2 V_{\text {eff }}\right|-2 L$.

\section{RESULTS}

Our solution involves six different phases. In fact, beyond the "pure" BW, SW, and CW phases and the hightemperature homogeneous $(\mathrm{H})$ state $(\Gamma=0=\Delta)$, there are intermediate phases which lack inversion symmetry; on them a BW order coexists with CW or SW order $(\Delta \neq 0 \neq \Gamma)$. The

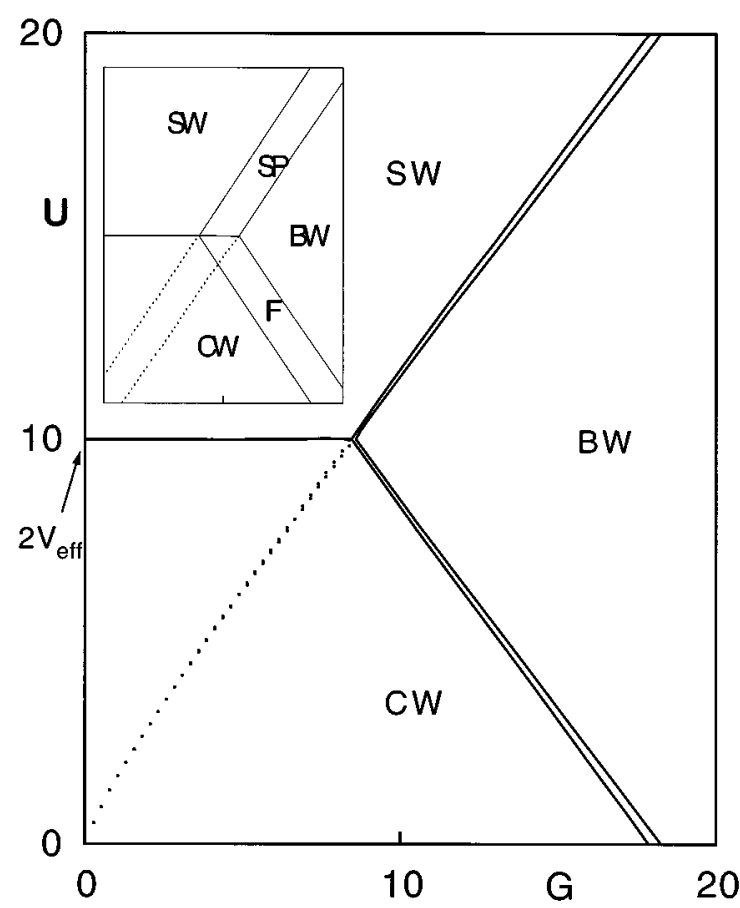

FIG. 1. Phase diagram in the plane $[G, U]$ for $V_{\text {eff }}=5$. The magnification shows the $\mathrm{F}$ and SP phases.

intermediate phase between BW and SW phases is a spinPeierls (SP) state, ${ }^{9,11}$ while that between BW and CW phases is ferroelectric $(\mathrm{F})$. The phase transitions $\mathrm{CW} \rightarrow \mathrm{F} \rightarrow \mathrm{BW}$ and $\mathrm{SW} \rightarrow \mathrm{SP} \rightarrow \mathrm{BW}$ are continuous, while the transition $\mathrm{CW}$ $\rightarrow \mathrm{SW}$ is discontinuous.

According to Eq. (4), at intermediate F or SP phases it holds that $\tau=-t D /[2 G(D+G)]$. The boundaries of these intermediate phases (e.g., BW-F or F-CW) are obtained by equating this expression with the value of $\tau$ at a "pure" phase.

\section{A. Phase diagram at $T=0$}

Let $D_{\mathrm{BW}}(G)<D<D_{\mathrm{CW}}(G)$ be the boundaries of the intermediate $\mathrm{F}\left(U<2 V_{\text {eff }}\right)$ or SP $\left(U>2 V_{\text {eff }}\right)$ phases. Reference 1 shows the curves $D_{\mathrm{BW}}(G)$ and $D_{\mathrm{CW}}(G)$. In the case $G \ll 2 \pi t$, the widths of the intermediate phases are exponentially small, holding that $D_{\mathrm{CW}} \approx D_{\mathrm{BW}} \approx-2 G^{2} /(\pi t+2 G)$ and $D_{\mathrm{CW}}-D_{\mathrm{BW}} \approx \pi t[G /(\pi t+2 G)]^{3} \exp [-2 \pi t / G]$. In the opposite limit, $G \gg 2 \pi t$, these boundaries are given by $D_{\mathrm{CW}}=-G+\sqrt[3]{\left[3 G t^{2} / 2\right.}$ and $D_{\mathrm{BW}}=-G+2 G t /(4 t+G)$.

In order to obtain a physical feeling of the stability range of the different phases, let us first consider a phase map in the plane $[G, U]$ for a fixed $V_{\text {eff }}$ (remember that $G=L+V_{\text {eff }}$ ). Figure 1 illustrates the case $V_{\text {eff }}=5$. Defining $f_{\mathrm{CW}}(G)=D_{\mathrm{CW}}(G)+2 G$, and an analogous expression for $f_{\mathrm{BW}}(G)$, we conclude the following:

(a) The $\mathrm{SW}$ phase is stable for $U>2 V_{\text {eff }}$ and $U>f_{\mathrm{CW}}(G)$.

(b) The $\mathrm{CW}$ phase is stable for $U<2 V_{\text {eff }}$ and $U<4 V_{\text {eff }}-f_{\mathrm{CW}}(G)$.

(c) The $\mathrm{BW}$ phase is stable for $4 V_{\mathrm{eff}}-f_{\mathrm{CW}}(G)$ $<U<f_{\mathrm{CW}}(G)$.

(d) Obviously, the ferroelectric and spin-Peierls phases correspond to the small strips lying between the CW-BW and BW-SW phases, respectively. 


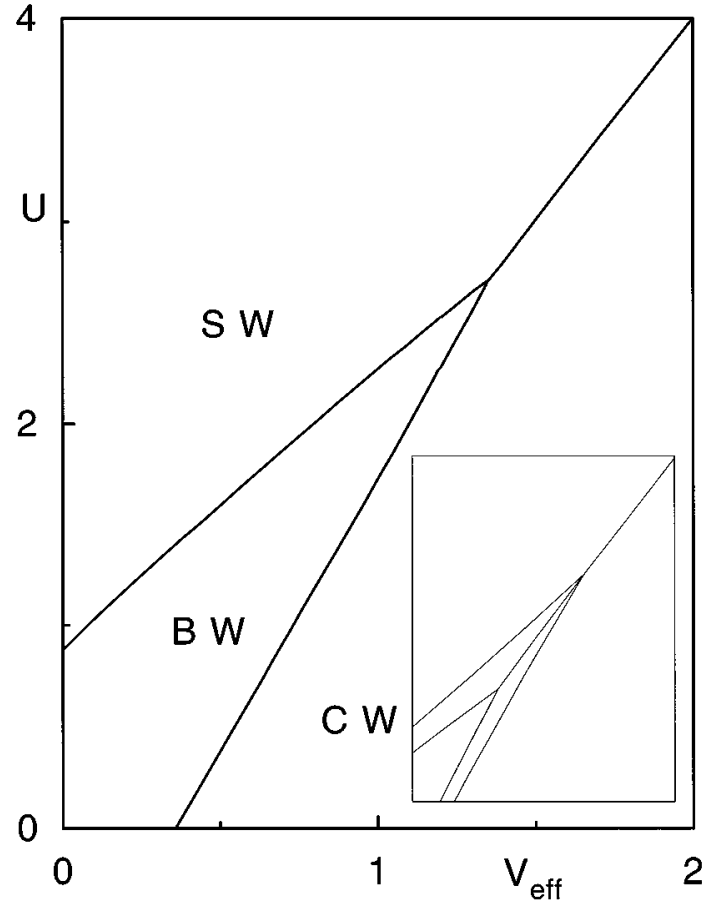

FIG. 2. Phase diagram in the plane $\left[V_{\text {eff }}, U\right]$ for $L=0.5$. Also, a magnification is provided to make apparent the intermediate phases.

In this way, the CW-F-BW and SW-SP-BW phase boundaries are related to each other by a mirror reflection into the horizontal line $U=2 V_{\text {eff }}$. Thus, the phase diagram can be obtained for any value of $V_{\text {eff }}$ by using the dotted curve of Fig. 1.

Our conclusions are consistent with Hirsch results; ${ }^{10}$; especially his condition for the BW loss, $U>U_{c}=4 g^{2} / K$, is a particularization of our results for the case $V=0=\lambda$, and a small $t$.

It is also instructive to consider the $T=0$ phase diagram in the plane $\left[V_{\text {eff }}, U\right]$, and a fixed value of $L$; the case $L=0.5$ is shown in Fig. 2. From these two figures and our mathematical analysis we conclude:

(i) An increase in $L$, all other parameters fixed, leads from a SW or CW to a BW phase; this result is obvious, since $L$ measures the relative importance of intercell $e$-ph interaction. The BW phase is precluded if $L$ is lower than a critical value, $L<L_{0}$. An approximate expression for $L_{0}$ (particularly suitable for small or moderate values of $V_{\text {eff }} / \pi t$ ) is $L_{0}=\sqrt{V_{\text {eff }}^{2}+\pi^{2} t^{2} / 4}-\pi t / 2$. For a fixed $L$, the upper boundary of the BW region (recall Fig. 2) is given by $U=U_{0} \sim 2 \sqrt{L^{2}+L \pi t}, V_{\text {eff }}=U_{0} / 2$.

(ii) Let us fix $L, V_{\text {eff }}$ and increase $U$. At $U=0$ the system is in a CW or BW-like $(\Delta \neq 0)$ phase (see Figs. 1 and 2). For $L>L_{0}$ an increase in $U$ leads to a continuous transition to the $\mathrm{SW}$ phase by crossing through the BW-like phases (e.g., $\mathrm{CW}$ $\rightarrow \mathrm{F} \rightarrow \mathrm{BW} \rightarrow \mathrm{SP} \rightarrow \mathrm{SW}$ ). If $L<L_{0}$, there is a direct and discontinuous $\mathrm{CW} \rightarrow \mathrm{SW}$ phase transition at $U=2 V_{\text {eff }}$.

(iii) We now fix $U, L$ and vary $V_{\text {eff }}$. For $V_{\text {eff }}=0$ and $L>L_{0}, U>f_{\mathrm{CW}}(L) \approx L[1+\pi t /(\pi t+2 L)]$, the system is in a SW phase, while lower values of $U$ correspond to BW. A first increase in $V_{\text {eff }}$ favors the BW phase, while a further increase leads to $\mathrm{CW}$. The $\mathrm{BW} \rightarrow \mathrm{F} \rightarrow \mathrm{CW}$ transition approximately occurs at $V_{\text {eff }}=[U+L] / 3$ for small $t$.

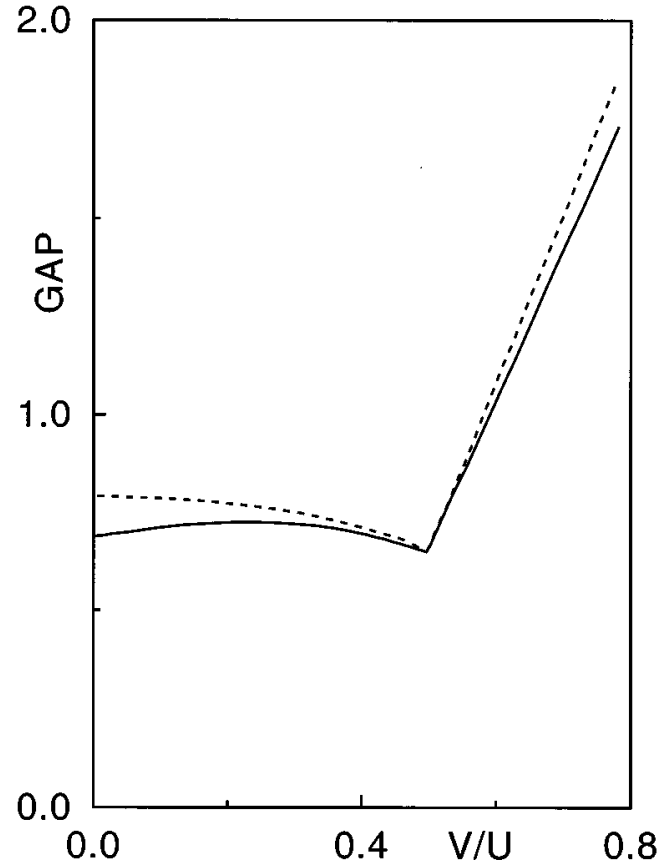

FIG. 3. Excitation gap $\mu_{g}$ vs $V$ for a four-atom cluster with $U=1$ and $g=\lambda=0$. The exact and HF results correspond to solid and dotted curves, respectively.

(iv) Finally we consider the effect of an increase in the electronic transfer term $t$ (e.g., due to an external pressure), which leads to a radial movement of point $(G / t, D / t)$ toward the origin in Fig. 1 of RG. In this case a SW-CW transition is precluded. A transition from $\mathrm{SW}$ or $\mathrm{CW}$ regions toward the $\mathrm{BW}$ phase is possible if $-G<D<0$, while $\mathrm{BW}$ is stable if $D<-G<0$. The SW or CW phases are stable if $D>0$.

\section{B. Effect of temperature}

Let us increase the temperature from $T=0$. According to $\mathrm{RG}$, if we start on a SW or CW phase, only a transition to the $\mathrm{H}$ phase is possible. The same is true for the BW phase, excepting in a narrow strip located around the intermediate $\mathrm{F}$ or SP phases and $G<5.7 t$. Over this strip we have the sequence of phase transitions $\mathrm{BW} \rightarrow \mathrm{SP} \rightarrow \mathrm{SW} \rightarrow \mathrm{H}$ or $\mathrm{BW} \rightarrow \mathrm{F}$ $\rightarrow \mathrm{CW} \rightarrow \mathrm{H}$ on increasing $T .{ }^{1}$ This phenomenon is very peculiar, since a symmetry reduction occurs on increasing temperature (as F or SP phases lack inversion symmetry, while BW phase has that symmetry). This result is not an "artifact" of HF approximation, since it persists even when $e-e$ repulsion is absent. Only a few other examples of this kind are known. ${ }^{15}$

It is necessary to point out that our analisys for $T \neq 0$ is more reliable for weak $e$-e correlation. ${ }^{1}$ Note also that Landau theorem forbids LRO in one-dimensional systems; thus, the concept of phase transition becomes blurred, ${ }^{6,8}$ and our LRO parameters must be replaced by short-range correlations. However, the correlation length is very large for $T<T_{c} / 4$; here $T_{c}$ is the mean field critical temperature. ${ }^{6}$

\section{Fermi gap}

Now we analyze the Fermi gap, defined by $^{3} \quad \mu_{g} \equiv \mu\left(N_{e}+1\right)-\mu\left(N_{e}\right)$, where $\mu\left(N_{e}\right)=E_{\mathrm{GS}}\left(N_{e}\right)$ 
$-E_{\mathrm{GS}}\left(N_{e}-1\right)$ is the chemical potential and $E_{\mathrm{GS}}\left(N_{e}\right)$ is the ground-state energy for a system with $N_{e}$ electrons. Postulating periodic HF solutions, it holds that $\mu_{g}$ coincides with the gap in the quasiparticles spectrum, $\delta_{g}$; the latter one is given by the minimum of $W_{k,+}-W_{k,-}=2 W_{k}$ in Eq. (3). However, $\mu_{g} \neq \delta_{g}$ for the exact solution of the interacting system. For example, in the $t \rightarrow 0, g=\lambda=0$ case, we have the exact results $\mu_{g}=U, \delta_{g}=U-V$ for the SW phase; $\mu_{g}=4 V-U$, $\delta_{g}=3 V-U$ for the $\mathrm{CW}$ phase.

Since $\tau>\Delta$, the HF gap lies at $k=\pi / 2$, holding that $\mu_{g}=2 \sqrt{4 G^{2} \Delta^{2}+A^{2} \Gamma^{2}}$. In the former case $(t \rightarrow 0$, $\lambda=g=0)$ the HF gap goes to $\mu_{g}=A$. The latter one reproduces the exact results for the chemical potential, but not the minimal quasiparticle excitation energy $\delta_{g}$.

\section{Test for the HF approximation}

Now we compare the exact and HF results for the gap $\mu_{g}$. Both calculations are done in a four-atom cluster; thus, finite-size effects affect $\mathrm{HF}$ and exact solutions in the same manner. Figure 3 shows $\mu_{g}$ versus $V$ for $U=1, t=0.2$, $g=\lambda=0$. The HF curve (dotted) closely follows the exact results (solid curve), especially for $U \sim 2 V$. However, the exact calculation leads to a local maximum of $\mu_{g}$ at
$V \sim 0.2 U$, while the HF phase gives a monotonic decrease of the gap when $0<V<U / 2$. The kink at $U=2 V$ is due to the SW-CW transition.

We have also compared the thermal behavior of exact and HF solutions using small clusters. ${ }^{1,4,15}$ For strong $e-e$ interactions we have concluded that the HF approximation is fair when $T<T_{c} / 4$ especially in the CW phase. However, the $\mathrm{HF}$ approximation fails to account the magnetic properties of the SW phase. ${ }^{4,16}$ In particular, for $g=\lambda=0, U>2 V, U$ $\gg t$, the Hubbard and antiferromagnetic Heisenberg models become equivalent, ${ }^{4}$ holding $J=2 t^{2} /(U-V)$ for the Heisenberg exchange. The HF approximation breaks the spin rotational symmetry, thus being inadequate to describe the magnetic correlations and low-energy excitations of the SW phase. $^{15}$

In the $t \rightarrow \infty$ limit, the exact and HF results converge, ${ }^{9}$ leading to the same exponential behavior for the gap of the one-dimensional Hubbard model, $\delta_{g} \propto \exp [-2 \pi t / U]$.

\section{ACKNOWLEDGMENTS}

This work was supported by FONDECYT (Project Nos. 1940341 and 1950655), FAPESP, FUNDUNESP, and CNPQ.
${ }^{1}$ J. Rössler and D. Gottlieb, J. Phys. Condens. Matter 2, 3723 (1990).

${ }^{2}$ N. Tomita and H. Fukutome, Solid State Commun. 81, 659 (1992).

${ }^{3}$ L. Lieb and F.Y. Wu, Phys. Rev. Lett. 20, 1445 (1968).

${ }^{4}$ J. Rössler, B. Fernández, and M. Kiwi, Phys. Rev. B 24, 5299 (1981).

${ }^{5}$ J. Hirsch, Phys. Rev. Lett. 53, 2327 (1984).

${ }^{6}$ P.A. Lee, T. Rice, and P.W. Anderson, Phys. Rev. Lett. 31, 462 (1973)

${ }^{7}$ E. Fradkin and J. Hirsch, Phys. Rev. B 27, 1680 (1983); 27, 4302
(1983).

${ }^{8}$ L. Degiorgi, G. Grüner, K. Kim, R.H. McKenzie, and P. Watcher, Phys. Rev. B 49, 14754 (1994).

${ }^{9}$ S. Kivelson and D. E. Heim, Phys. Rev. B 26, 4278 (1982).

${ }^{10}$ J. Hirsch, Phys. Rev. Lett. 51, 296 (1983).

${ }^{11}$ C. Esparza, M. Elgueta, and J. Rössler, J. Phys. Condens. Matter 6, 4361 (1994).

${ }^{12}$ J.C. Hicks and J. Tinka Gammel, Phys. Rev. B 37, 6315 (1988).

${ }^{13}$ A. Painelli and A. Girlando, Phys. Rev. B 48, 10683 (1993).

${ }^{14}$ P. Kopietz, Phys. Rev. B 48, 13789 (1993).

${ }^{15}$ D. Gottlieb and M. Lagos, Phys. Rev. B 39, 2960 (1989).

${ }^{16}$ C. Henríquez; M.Sc. thesis, Universidad de Chile, 1989. 